\title{
Partially unroofed coronary sinus with persistent left superior vena cava: the utility of two and three-dimensional transesophageal echocardiography
} -a case report-

\author{
Hiroshi Yonekura, Shinya Kanazawa, Ikuko Miyawaki, and Kazuo Yamazaki \\ Department of Anesthesiology and Critical Care, Kobe City Medical Center General Hospital, Kobe, Japan
}

Unroofed coronary sinus (URCS) is a rare cardiac anomaly, in which communication occurs between the coronary sinus (CS) and the left atrium (LA) because of partial or complete absence of the CS roof. A 30-year-old woman was scheduled for surgical closure of atrial septal defect, mitral valve repair and tricuspid annuloplasty. The intraoperative transesophageal echocardiography (TEE) revealed left-to-right shunt between the CS and the LA. The three-dimensional (3D) TEE confirmed the diagnosis of partially URCS. This defect was repaired with a pericardial patch. In this case, the 3D images of URCS, which were a helpful supplement to the $2 \mathrm{D}$ images, providing better visualization of the wall defect and more information regarding the size and location of the defect. The combined use of $2 \mathrm{D}$ and $3 \mathrm{D}$ images provides valuable information to aid in understanding the anatomy and morphology of this rare anomaly. (Korean J Anesthesiol 2014; 67: $52-56)$

Key Words: Congenital heart defects, Coronary sinus, Persistent left superior vena cava, Three-dimensional echocardiography.

Unroofed coronary sinus (URCS) is a rare cardiac anomaly, in which communication occurs between the coronary sinus (CS) and the left atrium (LA) because of partial or complete absence of the CS roof. This anomaly is strongly associated with a persistent left superior vena cava (PLSVC). In this report, we have described the occurrence of URCS in an adult cardiac surgery case, in which two- and three-dimensional transesophageal echocardiography (TEE) were collectively used to successfully determine the diagnosis and classification.

\section{Case Report}

A 30-year-old, previously healthy woman was referred to our facility after being diagnosed with atrial septal defect (ASD),

Received: May 14, 2013. Revised: July 6, 2013. Accepted: July 7, 2013.

Corresponding author: Hiroshi Yonekura, M.D., Department of Anesthesiology and Critical Care, Kobe City Medical Center General Hospital, 2-1-1, Minatojimaminamimachi, Chuo-ku, Kobe-city, Hyogo 650-0047, Japan. Tel: 81-78-301-4321, Fax: 81-78-302-7537, E-mail: komekurahiroshi@gmail.com

@This is an open-access article distributed under the terms of the Creative Commons Attribution Non-Commercial License (http:// creativecommons.org/licenses/by-nc/3.0/), which permits unrestricted non-commercial use, distribution, and reproduction in any medium, provided the original work is properly cited. 
moderate mitral regurgitation, and severe tricuspid regurgitation. Her past medical history was unremarkable. She complained of mild dyspnea on exertion. On physical examination, her heart rate was regular at 60 beats $/ \mathrm{min}$, and blood pressure was 110/50 mmHg. Heart and lung auscultation were normal except for a systolic murmur over the cardiac apex. The remainder of her physical examination was normal. Preoperative echocardiogram showed a sinus rhythm with an incomplete right bundle block and normal axis. Preoperative transthoracic echocardiography (TTE) revealed a dilated CS $(33 \mathrm{~mm})$ and an 18-mm defect in the posterior part of the LA with predominantly left-to-right shunting. An inferior sinus venosus ASD or a CS ASD was suspected. Because the CS was enlarged, the presence of a PLSVC was considered. The right atrium (RA) and right ventricle (RV) were dilated, but RV function was normal. The pulmonary veins were not well visualized. Calculation of the pulmonary-to-systemic blood flow ratio was estimated at $3.3: 1.0$. Contrast-enhanced chest tomography confirmed a PLSVC. The patient was scheduled for surgical closure of the ASD, mitral valve repair, and tricuspid annuloplasty.

In the operating room, routine monitoring devices and a radial arterial catheter were placed. After induction with midazolam, $0.1 \mathrm{mg} / \mathrm{kg}$, fentanyl, $4 \mu \mathrm{g} / \mathrm{kg}$, rocuronium, $0.6 \mathrm{mg} / \mathrm{kg}$, and tracheal intubation, anesthesia was maintained with $\mathrm{O}_{2}$ air combination, sevoflurane, remifentanil, and rocuronium. A pulmonary artery catheter was inserted from the right internal jugular vein without difficulty. The pulmonary arterial pressure was 22/10 mmHg. Thereafter, using an IE-33 ultrasound system with an X7-2t matrix (Philips Medical, Andover, MA, USA), a
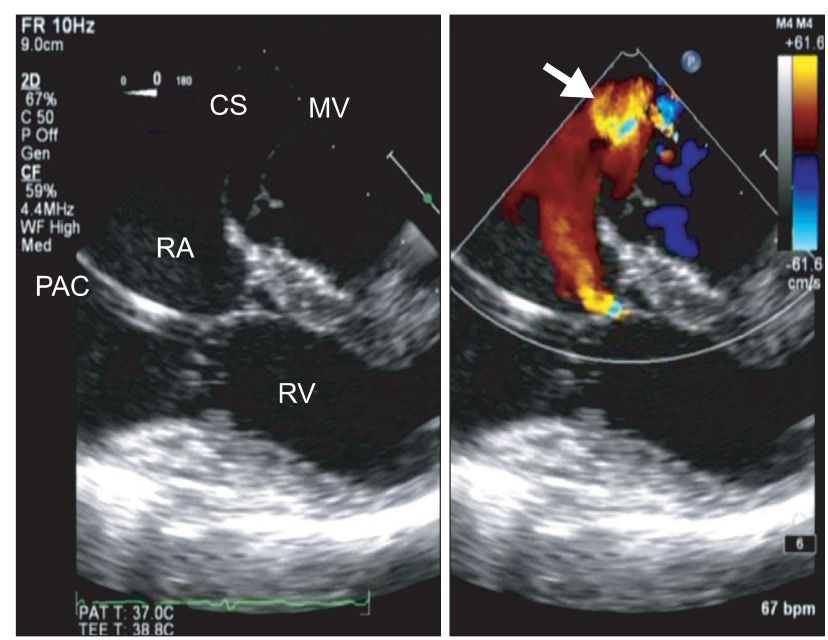

Fig. 1. In the midesophageal 4-chamber view of the CS, the wall closest to the left atrium (LA) appears to be missing, suggesting communication between the CS and the posterior LA wall. Color Doppler views demonstrate left-to-right shunting between the LA and the CS (arrow). CS: coronary sinus, MV: mitral valve, PAC: pulmonary artery catheter, RA: right atrium, RV: right ventricle.
TEE probe was inserted into the esophagus. The TEE confirmed preoperative echocardiographic findings of moderate mitral regurgitation and severe tricuspid regurgitation. In the midesophageal position and 0 degree scan plane, with the CS in the middle of the image, the ostium of the CS was dilated $(25 \mathrm{~mm})$ and the CS wall closest to the LA appeared to be missing, suggesting communication between the CS and the posterior LA wall. Color Doppler showed predominantly left-to-right shunting between the CS and the LA (Fig. 1). All pulmonary veins emptied into the LA. Contrast study was performed by injecting agitated saline into the left antecubital vein. Bubble contrast was observed in the CS before appearing in the right heart, thus confirming the diagnosis of PLSVC. The remainder of the septum was intact. After optimizing a midesophageal view of the CS at 0 degrees, the three-dimensional (3D) full-volume mode was selected and the region of interest was adjusted to include both the CS and the surrounding tissues. The 3D full-volume view showed that the CS wall was partially unroofed. The defect was located at the midportion of the CS (Fig. 2). The 3D images confirmed the diagnosis of partially URCS.

After systemic heparinization, the venae cavae, the PLSVC, and the ascending aorta were cannulated, and cardiopulmonary bypass was established. During surgical exploration, a defect from the CS into the LA was identified at the posterior LA wall, consistent with pre-CPB TEE findings. This defect was repaired with a pericardial patch, allowing the CS effluent to drain normally into the RA. The patient underwent uneventful mitral valve repair and tricuspid annuloplasty and was easily weaned off bypass with vasopressor and inotropic support (norepineph-

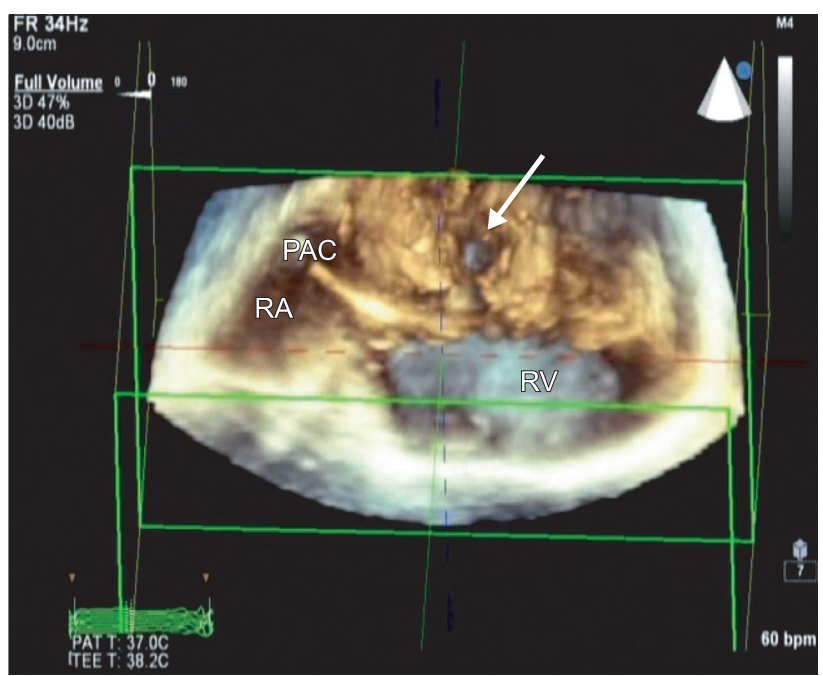

Fig. 2. The 3D full-volume image shows the partially unroofed coronary sinus. The defect is located in the midportion of the CS. CS: coronary sinus, PAC: pulmonary artery catheter, RA: right atrium, RV: right ventricle. 

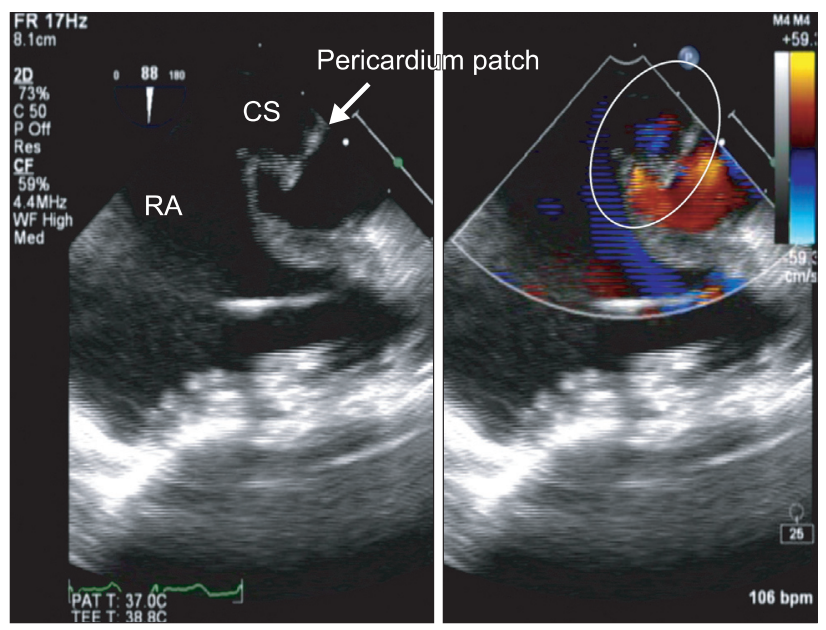

Fig. 3. After surgical repair, no residual shunt across the CS is apparent (circle). CS: coronary sinus, RA: right atrium.

rine; $0.05 \mu \mathrm{g} / \mathrm{kg} / \mathrm{min}$, dobutamine; $3 \mu \mathrm{g} / \mathrm{kg} / \mathrm{min}$ ).

Post-CPB TEE findings showed no residual shunt across the CS (Fig. 3). After surgery, she has not suffered from any electrophysiological conduction abnormalities. The trachea was extubated uneventfully in the intensive care unit within $10 \mathrm{~h}$, and the patient recovered without any apparent complications.

\section{Discussion}

The CS is located posteriorly in the left atrioventricular groove and opens into the RA through the orifice, which is located in the posterior and inferior RA walls and superior to the tricuspid valve [1]. Normally there is no direct communication between the CS and the LA. URCS is a rare cardiac anomaly, first described by Raghib et al. [2] in 1965. In this anomaly, communication occurs in the wall between the CS and the LA because of partial or complete absence of the CS roof. The ostium of the CS is frequently large because of the increased flow.

This anomaly is strongly associated with a PLSVC, atypically draining the left internal jugular and subclavian veins into the CS. A PLSVC occurs in $0.1-0.5 \%$ of the general population, with $8 \%$ draining into the LA [3]. In the series described by Quaegebeur et al. [4], 75\% (18/24) cases of URCS were associated with a PLSVC. The morphologic types of URCS were classified in the following four types by Kirklin and Barratt-Boyes [4] according to the partial or complete absence of the CS roof, location of the defect, and presence of PLSVC: type I: completely URCS with PLSVC; type II: completely URCS without PLSVC; type III: partially unroofed midportion of CS; and type IV: partially unroofed terminal portion of CS. Surgical management depends on the specific morphology of the lesion.

The preoperative diagnosis of URCS is important but often difficult because of nonspecific clinical features. This anomaly should be suspected in patients with a PLSVC and a history of paradoxical embolism or brain abscess. Precise preoperative diagnosis and classification are often difficult because of complex anatomy and other cardiac anomalies. Cardiac angiography, multislice computed tomography, cardiac magnetic resonance imaging, and echocardiography (TTE or TEE) are helpful for obtaining preoperative information related to URCS [5-7]. Xie et al. [7] reported 20 cases of URCS, in which diagnostic accuracy of echocardiography was $65 \%$ and accuracy of URCS types was $84.6 \%$. They concluded that a combination of TTE and contrast echocardiography can result in an accurate diagnosis and URCS classification. They also suggested that additional attention must be given to the possibility of URCS in the following situations: (i) ASDs (ostium primum and sinus inferior venous), (ii) complex congenital heart disease, (iii) CS dilation, and (iv) inability to detect normal CS draining into the RA in multiple sectional views. TTE is the most widely used imaging modality for congenital heart disease. However, it is limited in its ability to visualize posterior cardiac structures, such as CS and LA. TEE is more accurate in visualizing these posterior cardiac structures and provides better delineation of anatomy and size of the defect. On TEE, URCS may be seen in the midesophageal two-chamber or long-axis views [8,9]. When URCS is present, the CS wall closest to the LA appears to be missing, suggesting communication between the posterior aspect of the LA and the CS [8]. The presence of a PLSVC can be determined by TEE after injecting agitated saline into a patient's left antecubital vein and assessing for the presence of saline bubbles in the CS before their appearance in the right heart.

Anesthetic management for patients with URCS is same as that of ASD. The magnitude and direction of shunt, and the presence of pulmonary hypertension are important for the management during anesthesia. Anesthetic management goals are to maintain heart rate, contractility, and preload to maintain cardiac output. An increase pulmonary vascular resistance may result in production of a right (R)-to-left (L) shunt and lead to hypoxemia. Hemodynamic maintenance will be achieved through a reduction of the L-to- $\mathrm{R}$ shunt. The risk of paradoxical emboli through venous accesses (air bubbles, microaggregates) is always present. Intraoperative benefits from TEE include not only diagnosis and classification as mentioned before, but also recognizing the adequacy of the repair, intracardiac air, and ventricular volume and function.

Surgical repair of an URCS depends on the presence or absence of a PLSVC. Without an associated PLSVC, the CS ostium is patched from the RA, and the CS is left to drain into the LA, resulting in a clinically insignificant $(<5 \%)$ right-to-left shunt. When a PLSVC is present, other techniques should be used because a simple patch closure would allow CS blood and venous 


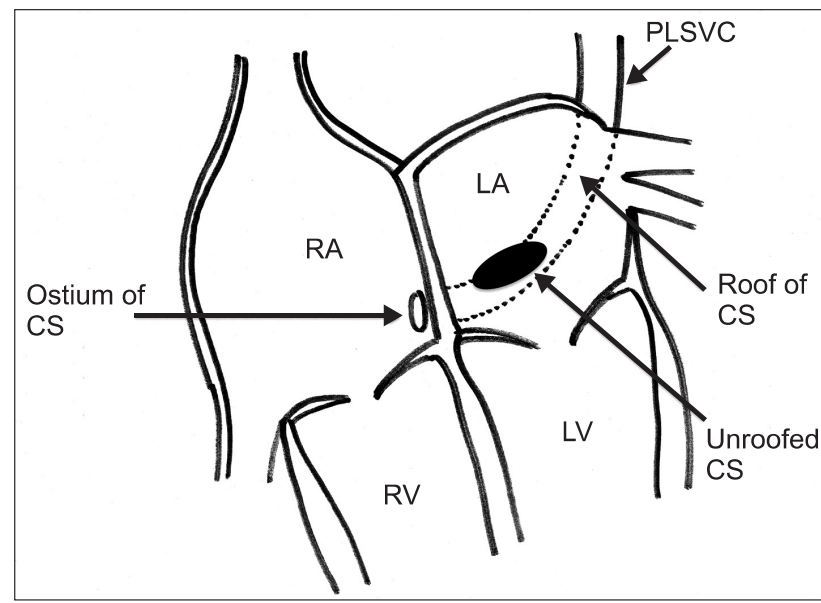

Fig. 4. A schematic view of this case. The morphologic type of unroofed coronary sinus (URCS) is type III, with a partially unroofed coronary sinus midportion with PLSVC. CS: coronary sinus, LA: left atrium, LV: left ventricle, PLSVC: persistent left superior vena cava, RA: right atrium, RV: right ventricle.

return from the PLSVC to enter the LA, resulting in an unacceptable degree of cyanosis. The PLSVC may be ligated if an innominate vein is present, or it can be transected and sewn to the left pulmonary artery, or it can be rerouted directly to the RA [4]. The roof of the defect (in the LA) is most commonly covered with the pericardium, and the CS is allowed to drain normally into the RA. This is often referred to as a baffle. There are multiple options for URCS repair. Ultimately, the technique for surgical repair of URCS will depend on the specific morphology of the individual lesion.
By obtaining the 3D volumetric imaging and using the cropadjust function, we were able to visualize the detailed anatomical structures. The 3D TEE can provide clear images of near-field structures such as an atrial septum. It has been reported to offer advantages in terms of anatomic relationships of ASDs with surrounding structures in a single view $[10,11]$. The availability of 3D TEE for URCS has been recently documented in a few case reports [12-14], which states that 3D images provide more precise anatomic and morphological information for diagnoses. In this case, 2D images were not sufficient for diagnosis and classification because they could not show the precise anatomical location of the defect. By obtaining 3D images, the wall defect can be adequately visualized and more information regarding its size and location can be obtained. Further, we confirmed the morphologic type of URCS as type III (partially unroofed midportion of CS with PLSVC) (Fig. 4). Before surgical exploration, we were able to get more anatomical and morphological information on URCS, this information was useful for surgical decision making for URCS repair. The surgical technique involved using a pericardial patch on the roof of the defect of the LA, and the CS was left to drain into the RA.

In summary, we have described a rare URCS with a PLSVC in an adult cardiac surgery case. We also described the 3D images of URCS, which were a helpful supplement to the 2D images, providing better visualization of the wall defect and more information regarding the size and location of the defect. Our images clearly demonstrated the anatomic structures of this anomaly. Thus, we believe that the combined use of 2D and 3D images provides valuable information to aid in understanding the concept of this rare anomaly.

\section{References}

1. Adatia I, Gittenberger-de Groot AC. Unroofed coronary sinus and coronary sinus orifice atresia. Implications for management of complex congenital heart disease. J Am Coll Cardiol 1995; 25: 948-53.

2. Raghib G, Ruttenberg HD, Anderson RC, Amplatz K, Adams P Jr, Edwards JE. Termination of left superior vena cava in left atrium, atrial septal defect, and absence of coronary sinus: a developmental complex. Circulation 1965; 31: 906-18.

3. Ootaki Y, Yamaguchi M, Yoshimura N, Oka S, Yoshida M, Hasegawa T. Unroofed coronary sinus syndrome: diagnosis, classification, and surgical treatment. J Thorac Cardiovasc Surg 2003; 126: 1655-6.

4. Quaegebeur J, Kirklin JW, Pacifico AD, Bargeron LM Jr. Surgical experience with unroofed coronary sinus. Ann Thorac Surg 1979; 27: 41825.

5. Thangaroopan M, Truong QA, Kalra MK, Yared K, Abbara S. Images in cardiovascular medicine. Rare case of an unroofed coronary sinus: diagnosis by multidetector computed tomography. Circulation 2009; 119: e518-20.

6. Hahm JK, Park YW, Lee JK, Choi JY, Sul JH, Lee SK, et al. Magnetic resonance imaging of unroofed coronary sinus: three cases. Pediatr Cardiol 2000; 21: 382-7.

7. Xie MX, Yang YL, Cheng TO, Wang XF, Li K, Ren PP, et al. Coronary sinus septal defect (unroofed coronary sinus): echocardiographic diagnosis and surgical treatment: Int J Cardiol 2013; 168: 1258-63.

8. Joffe DC, Rivo J, Oxorn DC. Coronary sinus atrial septal defect. Anesth Analg 2008; 107: 1163-5.

9. Huang XS. Images in cardiovascular medicine. Partially unroofed coronary sinus. Circulation 2007; 116: e373.

10. Johri AM, Passeri JJ, Picard MH. Three-dimensional echocardiography: approaches and clinical utility. Heart 2010; 96: 390-7. 
11. Johri AM, Witzke C, Solis J, Palacios IF, Inglessis I, Picard MH, et al. Real-time three-dimensional transesophageal echocardiography in patients with secundum atrial septal defects: outcomes following transcatheter closure. J Am Soc Echocardiogr 2011; 24: 431-7.

12. Kam KK, Looi JL, Yip YY, Lee AP, Wong JK, Yu CM. Unroofed coronary sinus on real-time three-dimensional transesophageal echocardiography. Int J Cardiol 2013; 164: e13-4.

13. Singh A, Nanda NC, Romp RL, Kirklin JK. Assessment of surgically unroofed coronary sinus by live/real time three-dimensional transthoracic echocardiography. Echocardiography 2007; 24: 74-6.

14. Vargas-Barrón J, Espinola-Zavaleta N, Romero-Cárdenas A, Roldán FJ, Keirns C, Hernández-Reyes P, et al. Two- and three-dimensional echocardiographic unroofed coronary sinus. J Am Soc Echocardiogr 2001; 14: 742-4. 